\title{
Clinical Analysis of Nausea and Vomiting in Patients with Gastrointestinal Diseases
}

\author{
Ling Zhang \\ Luohe Medical College, Luohe, Henan, 462000
}

Keywords: gastroenterology; nausea and vomiting; cause; diagnosis; treatment

\begin{abstract}
Objective: To analyze the causes of nausea and vomiting in patients with digestive diseases, enhance the understanding of nausea and vomiting caused by medical diseases, and provide evidence for clinical diagnosis to avoid missed diagnosis and misdiagnosis. Methods: A retrospective analysis of 70 cases of hospitalized patients with nausea and vomiting as the main symptom in the Department of Gastroenterology, Second Affiliated Hospital of Luohe Medical College from August 2014 to March 2016. Results: Of the 70 patients, chronic gastritis was the most common, accounting for $18.57 \%$, followed by acute gastroenteritis, accounting for $15.71 \%$. After targeted treatment of confirmed patients, the total effective rate is $80 \%$. Conclusion: The cause of nausea and vomiting in patients with gastroenterology is complicated, and the characteristics are $80 \%$ atypical. Clinicians should establish good diagnostic ideas and treatment methods.
\end{abstract}

\section{Introduction}

Nausea and vomiting are common symptoms in the clinical department of gastroenterology and one of the common causes of patient visits. The causes are different and complex. Except for a few patients with typical medical history, symptoms and signs, they can quickly and clearly diagnose, and a considerable proportion of patients have difficulty in identifying the cause and receiving timely treatment in a short period of time, and sometimes missed diagnosis and misdiagnosis. happened. Now, from the Department of Gastroenterology, Second Affiliated Hospital of Luohe Medical College, from August 2014 to March 2016, 70 cases of hospitalized cases with nausea and vomiting as the main symptoms were continuously selected for analysis.

\section{Data and Methods}

General Information among the 70 patients with digestive medicine, 32 were male and 38 were female. The maximum age is 92 years old, the minimum age is 17 years old, and the average age (58.97 \pm 20.33$)$ years old.

Diagnostic methods According to the patient's different medical history, symptoms and signs, give targeted examinations, such as blood, urine, fecal routine, blood biochemistry, B-ultrasound, $\mathrm{X}$-ray, CT, endoscopy and biopsy, etc. The diagnosis basis is shown in Table 1.

Treatment methods according to 70 cases of gastrointestinal vomiting in patients with gastroenterology, proton pump inhibitors are given to inhibit gastric acid secretion, timely rehydration to maintain the body's $\mathrm{pH}$, water, electrolyte balance, appropriate vitamin supplements, and appropriate treatment according to the original disease, Such as targeted administration of related anti-emetic drugs, antibiotic drugs, prokinetic drugs.

Efficacy judgment according to the clinical manifestations of patients before and after treatment to determine its therapeutic effect 1 markedly: after treatment, nausea and vomiting symptoms were significantly less than before treatment; 2 effective: after treatment, nausea and vomiting was less than before treatment; 3 invalid: The degree of nausea and vomiting did not decrease or even worse after treatment.

Statistical methods Survey data are entered into the computer to establish a database. The data were statistically analyzed using the SPSS 17 . U software package. The X-test was used for the 
count data. $\mathrm{P}<0.05$ was considered statistically significant.

\section{Results}

The cause of nausea and vomiting the clinical data of 70 patients with gastrointestinal vomiting can be seen that the proportion of vomiting caused by digestive diseases is $80.00 \%$, which is the main cause of vomiting in patients, among which chronic gastritis is the most common. The prevalence of non-digestive diseases accounted for $20.0 \%$, and the incidence of diabetic gastroparesis was more common, accounting for $5.71 \%$.

Gender distribution Among the 70 patients with vomiting admitted to the Department of Gastroenterology, 32 were male and 38 were female. The proportion of men with digestive diseases was $48.21 \%$, and that of women was $51.79 \%$. Among them, intestinal obstruction, abdominal malignant tumor, peptic ulcer and esophagitis were more common in male patients; the proportion of males with non-digestive diseases was $35.71 \%$, female is $64.29 \%$.

Age distribution and number of cases of different age groups The average age of patients with digestive diseases is $(60.00 \pm 20.40$ years old, the average age of patients with non-digestive diseases is (56. $14 \pm 20.57$ ) years old In the non-digestive system diseases, 4 cases of diabetic gastroparesis (63 years old, 65 years old, 66 years old, 85 years old), 2 cases of pregnancy (20 years old, 23 years old), 1 case of postoperative gastric fistula (77 years old), adrenal cortex 1 case (62 years old), 1 case (63 years old), 1 case (68 years old) with acute pharyngitis, 2 cases (54 years old, 71 years old) with cervical spondylosis, 1 case (24 years old) with snoring, cerebral infarction 1 Example (45 years old).

Results of the results of the treatment of 70 patients with clinical symptomatic treatment after the clinical total effective rate (effective + effective) was $80.00 \%$

\section{Discussion}

The causes of nausea and vomiting are extensive, including many factors, involving almost every system. According to the rapid onset of acute and chronic, acute vomiting is acute, often accompanied by abdominal pain, the history is relatively clear, easy to determine the cause; cause more causes of chronic vomiting, such as reflux esophagitis, pyloric obstruction, pregnancy, intestinal movement Disorders, central nervous system diseases, or systemic diseases, etc., accompanied by symptoms of acid reflux, heartburn, difficult to identify the cause, easily lead to missed diagnosis and misdiagnosis of the group. Press hair

The disease mechanism can also be divided into the following categories: reflex vomiting; central vomiting; vestibular vomiting; neurological vomiting. This study analyzed the clinical etiology of 70 patients with nausea and vomiting in the Department of Gastroenterology. The most common disease in the digestive system was diffuse gastritis (18.57\%), followed by acute gastroenteritis (15.71\%). However, non-digestive diseases also accounted for a certain proportion (20. 00\%), mainly endocrine diseases (8.53\%), of which diabetic gastroparesis is more common (5.71\%), suggesting that clinicians are diagnosed with nausea and vomiting. When it is the main manifestation of the disease, it is necessary to pay attention to the presence of non-digestive diseases, especially endocrine diseases. The study also showed that the proportion of male and female patients with nausea and vomiting as the main performance was relatively average (48.21\% for males and $51.79 \%$ for females). The proportion of males and females was also balanced. In the non-digestive system diseases, the proportion of male and female patients is relatively obvious (male $35.71 \%$, female 64.29\%), specific research on various causes, the difference is also more significant, such as diabetic gastroparesis, pregnancy, cervical vertebrae Both the disease and acute pharyngitis are female. Postoperative gastroparesis, snoring, cerebral infarction, adrenal insufficiency, and hyperthyroidism are male, indicating that clinicians cannot ignore the effects of gender in the process of receiving patients and treatment.

Analysis of patients with digestive system disease in this case, the incidence of patients aged 20 to 40 did not change significantly, the probability of disease in patients aged 40 to 60 years 
increased gently, the increase in the age of 60 to 80 years old, the vomiting patients in this age group should focus on Pay attention to the etiology of the digestive system. The decline in the prevalence of patients over 80 years of age may be related to the length of life. Among patients with non-digestive diseases, vomiting was diagnosed as early pregnancy in 2 young women, suggesting that vomiting in young women should be considered more. There were 4 cases of diabetic gastroparesis, all of which occurred after 50 years old; 2 cases of cervical spondylosis, 1 case of postoperative gastric fistula, 1 case of adrenal insufficiency, 1 case of hyperthyroidism and 1 case of acute pharyngitis. The age of onset was 55. More than one year old. The age distribution characteristics are very helpful for clinicians to diagnose, differentially diagnose and treat such diseases. Young doctors cannot ignore their importance.

When treating diseases with nausea and vomiting as the main symptoms, nausea and vomiting is only accompanied by symptoms. It is necessary to clearly identify the cause of nausea and vomiting, so that targeted treatment can be taken. Before the cause is confirmed, anti-infective drugs and strong anti-inflammation should not be blindly given. Drugs, etc., otherwise affect the treatment effect and delay the patient's condition, and eventually lead to the deterioration of the condition. At the same time, patients who are calling for emergency treatment due to nausea and vomiting should also pay enough attention to the clinic. Yan Liying's research shows that attaching importance to nausea and vomiting to call emergency patients is of great significance for the rescue of patients. Therefore, when the patient is admitted to hospital, the patient's disease history should be inquired in detail, and the patient's pathogenesis and characteristics of the disease should be known in time. At the same time, detailed laboratory examinations and auxiliary examinations must be performed according to the basic conditions of the patient's body in order to be treated. In the process, the corresponding treatment measures are taken, and the clinical treatment effect of the patient is closely monitored, and finally a clear diagnosis is made, and only in this way can a better therapeutic effect be achieved. In this study, 70 patients with nausea and vomiting in the Department of Gastroenterology were treated with symptomatic treatment after diagnosis. The total clinical effective rate was as high as $80.00 \%$. The therapeutic effect was ideal, indicating that the cause of the disease is a key factor in the treatment effect, which should cause the majority of medical work. The person attaches great importance to it. But there is still research 20. 00\% of patients have ineffective clinical treatment, so medical staff should closely monitor the clinical treatment effect of patients. If no significant effect is obtained, doctors should actively seek the cause of the disease and avoid overuse and abuse of the drug.

In summary, nausea and vomiting is a common symptom in patients with digestive medicine, and the causes of the disease are also complicated. The clinicians of digestive medicine often pay attention to endoscopic imaging and laboratory examination, and lack of excavation and clinical comprehensive analysis of medical history. Therefore, in the clinical diagnosis of nausea and vomiting in patients with gastroenterology, it is easy to make empirical errors. If you do not pay enough attention to such diseases, you often ignore the important details such as the patient's age, gender, physical signs and the efficacy after symptomatic treatment. Missed diagnosis, misdiagnosis occurred. The clinician should learn from the experience. For nausea and vomiting related diseases, detailed medical history, careful diagnosis, combined with diagnostic knowledge and related examinations should be analyzed to summarize the clinical characteristics of patients, and targeted treatment and strict monitoring and treatment. The effect is to improve the diagnosis and treatment ability of such diseases, and lay a solid foundation for future clinical work.

\section{References}

[1] Tang Rongjin. Clinical analysis of 78 patients with nausea and vomiting in patients with gastroenterology $[\mathrm{J}]$. Chinese practical medicine,

2013, 18: 138-139.

[2] Zhu Langchao, Jiang Xiling, Wang Xueling. Analysis of the causes of nausea and vomiting in 128 patients with gastroenterology [J]. Huaxi Medical Journal, 2010, 25(1): 119-120. 
[3] Yan Liying, Zhao Jing. Analysis of the condition of 75 patients with emergency vomiting and vomiting [J]. Jilin Medical Journal, 2013, 34 (31): 6540. 